Check for updates

Cite this: RSC Adv., 2018, 8, 30582

\title{
Pure exciplex-based white organic light-emitting diodes with imitation daylight emissions $\uparrow$
}

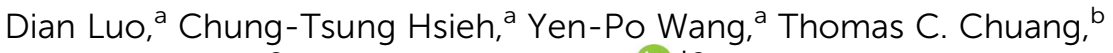 \\ Hsin-Hua Chang ${ }^{c}$ and Chih-Hao Chang (D) *a
}

An exciplex could be formed by blending a selected hole-transporting material (HTM)/electrontransporting material (ETM) pair, and the corresponding energy band gap is roughly determined by the energy difference between the lowest unoccupied molecular orbital (LUMO) of the ETM and the highest occupied molecular orbital (HOMO) of the HTM. In this study, three HTM/ETM combinations are adopted to generate blue, green, and red exciplexes, allowing us to design precise device architectures for the fabrication of exciplex-based white OLEDs (WOLEDs) with daylight-like emissions. The CIE coordinates of this WOLED varied close to the Planckian locus as the biases increase, with a high color rendering index of about 96. This high performance suggests this exciplex-based WOLED can provide high-quality white-light illumination. Photoluminance and lifetime measurements of the exciplex behavior of the HTM/ETM combinations indicate that the HTM and ETM selected should possess higher triplet energy bandgaps than those of their corresponding exciplex to avoid energy loss.

Received 11th June 2018

Accepted 24th August 2018

DOI: $10.1039 / \mathrm{c} 8 \mathrm{ra0} 4986 f$

rsc.li/rsc-advances excited state and the first singlet excited state $\left(\Delta E_{\mathrm{ST}}\right)$, allowing for the collection of triplet excitons via reverse intersystem crossing. ${ }^{1-3}$ Similarly, a small $\Delta E_{\mathrm{ST}}$ can be achieved with an exciplex generated by through-space intermolecular charge transfer between a hole-transporting material (HTM) and an electron-transporting material (ETM). ${ }^{4-6}$

The exciplex could be easily formed by applying commercially available materials. ${ }^{\mathbf{1 0 , 1 1}}$ Exciplex-based OLEDs can also be very efficient given appropriate HTM and ETM in the emitting layer (EML). In general, an exciplex could be generated using a bilayer structure with both HTM and ETM or by directly mixing an HTM and an ETM. The latter typically results in greater exciplex production yields. In principle, since the exciplex energy band gap is determined by the energy-level difference, the energy band gap could be adjusted by choosing appropriate materials. For instance, the energy band gap could be determined by the energy difference between the lowest unoccupied molecular orbital (LUMO) of the ETM and the highest occupied molecular orbital (HOMO) of the HTM. ${ }^{7-13}$

OLEDs which can effectively imitate daylight would be of particular use in high-latitude areas where sunlight exposure is highly seasonal. ${ }^{\mathbf{1 4 - 1 6}}$ In 2009, Jou et al. proposed a sunlight-style OLED with a correlated color temperature (CCT) tunable from 2300 to $12000 \mathrm{~K}$, proving the possibility of fabricating a new low color temperature light source. ${ }^{17}$ In 2013 , the same group reported OLEDs with a candle light-style yellowish orange chromaticity with a power efficiency of $29 \mathrm{~lm} \mathrm{~W}^{-1}$ at $100 \mathrm{~cd} \mathrm{~m}^{-2}$, a respective color rendering index (CRI) of 87 and a CCT of 2050 K. ${ }^{18}$ The fabricated device minimized potential harmful effects of excessive blue emission on the human eye. In 2014, they
${ }^{a}$ Department of Electrical Engineering, Yuan Ze University, Chung-Li, Taiwan 32003. E-mail: chc@saturn.yzu.edu.tw; Fax: +886 34638800 ext. 7517; Tel: +886 34514281 ${ }^{b}$ Department of Information Science and Technology, Vanung University, Chung-Li, Taiwan 32061

${ }^{c}$ Department of Aeronautical and Opto-Mechatronic Engineering, Vanung University, Chung-Li, Taiwan 32061

$\dagger$ Electronic supplementary information (ESI) available. See DOI: 10.1039/c8ra04986f 
demonstrated the feasibility of creating indoor sunset hues using a dusk-light-style OLED that exhibits a CCT ranging between 1500 and $3000 \mathrm{~K} .{ }^{19}$ The artificial dusk hue shows a 92 color rendering index at $2745 \mathrm{~K}$ with an $87 \%$ luminance spectrum resemblance with the sunset hue. However, all these devices used the host-guest system in the EML, which not only requires expensive molecular emitters but also suffers from different rates of degradation of the emitters. Herein, we propose a pure exciplex-based white OLED (WOLED) with a delicate device architecture design which can generate emissions that approximate daylight, where the WOLED emission color closely tracks the Planckian locus. ${ }^{20,21}$

\section{Experimental}

\section{OLED fabrication and characterization}

The multilayer OLED devices were fabricated on glass substrates which had the typical structure with the organic layers sandwiched between a bottom ITO $(120 \mathrm{~nm})$ anode and a top metal cathode. After routine ultra-sonication cleaning of the indium tin oxide (ITO) coated glass in deionized water and organic solvents, the ITO-coated glass was pre-treated with UVozone for 5 minutes. The organic and metal layers were deposited onto ITO substrate by thermal evaporation in a vacuum chamber with a base pressure of about $10^{-6}$ torr. Device fabrication was completed in a single cycle without breaking the vacuum. The active area was defined by a shadow mask $\left(2 \times 2 \mathrm{~mm}^{2}\right)$. Current density-voltage-luminance $(J-V-L)$ characterization was measured using a Keithley 238 current source-measure unit and a Keithley 6485 pico-ammeter equipped with a calibrated Si-photodiode. The electroluminescent spectra were recorded using an Ocean Optics spectrometer.

\section{Photophysical measurements}

Thin films for photophysical characterization were prepared by thermal evaporation on quartz substrates in a vacuum chamber. The fluorescence spectra, phosphorescence spectra, and lifetime measurements were characterized by using a fluorescence spectroscopy (HORIBA-fluoromax plus). Phosphorescence spectra of thin films or dilute solution (toluene) were conducted at $77 \mathrm{~K}$ by the spectrometer equipped with a microsecond flash Xe lamp as the pulsed excitation source. Different delay timescales were inserted between the pulsed excitation to collect the emission spectra. Time-resolved PL (TRPL) was measured by monitoring the decay of the intensity at the PL peak wavelength using the time-correlated single-photon counting technique with a nanosecond pulsed light-emitting diode $(320 \mathrm{~nm})$.

\section{Results and discussion}

\section{Device architecture designs for exciplex-based OLEDs}

To demonstrate our idea, all selected materials for the tested devices used commercially available compounds. Two commonly used HTMs, 4, $4^{\prime}, 4^{\prime \prime}$-tris $(N$-3-methylphenyl- $N$-phenylamino)triphenylamine ( $m$-MTDATA) ${ }^{22}$ and $4,4^{\prime}, 4^{\prime \prime}$-tris(carbazol9-yl)-triphenylamine (TCTA), ${ }^{23}$ were respectively selected as hole transport layer or the HTM for the EMLs. In addition, $3,5,3^{\prime}, 5^{\prime}$-tetra( $m$-pyrid-3-yl)-phenyl $\left[1,1^{\prime}\right]$ biphenyl $\quad$ (BP4mPy), ${ }^{24}$ 1,3,5-tris[(3-pyridyl)phen-3-yl]benzene (TmPyPB), ${ }^{25}$ and 4,6bis(3,5-di(pyridin-3-yl)phenyl)-2-methylpyrimidine

(B3PyMPM $)^{\mathbf{2 6}}$ are used as the electron transport layer or the ETM in the EMLs. Three combinations of HTM and ETM were used to produce an exciplex which was responsible for the blue to red emission shift. Since the energy band gap of exciplex is determined by the energy difference between the LUMO of the ETM and the HOMO of the HTM. Therefore, the emission color of the exciplex could be roughly estimated according to their energy levels before device fabrication. According to previous literature, the LUMO of BP4mPy, TmPyPB, and B3PyMPM were estimated to be around $2.57 \mathrm{eV}, 2.73 \mathrm{eV}$, and $3.20 \mathrm{eV}$, respectively. ${ }^{24-26}$ In addition, the respective HOMO of TCTA and $m$ MTDATA were estimated to be around $5.80 \mathrm{eV}$ and $5.10 \mathrm{eV}^{22,23}$ Thus, the respective LUMO/HOMO differences for the following combinations are: $4.09 \mathrm{eV}$ for TCTA : BP4mPy, $2.37 \mathrm{eV}$ for $m$ MTDATA : TmPyPB, and $1.90 \mathrm{eV}$ for $m$-MTDATA : B3PyMPM. They then could generate blue, green, and red emissions, respectively.

On the other hand, to imitate daylight emissions, the main carrier recombination zone was set at the red EML, producing an intense red emission at lower biases. In addition, a proper device architecture design will allow the carrier recombination zone to progressively extend to the green and blue EMLs as the forward bias increases. Hence, one could expect that the emission color of OLEDs might vary from red to white. Furthermore, a wide energy bandgap material, 2,6-bis(3-(9H-carbazol-9-yl)phenyl)pyridine (26DCzppy), ${ }^{27}$ with bipolar transport capability was inserted between the green and red EMLs to ensure the recombination zone formed at the red EML at a lower bias. This buffer layer could help adjust the carrier transport and the exciton diffusion. ${ }^{28}$

Two kinds of exciplex-based WOLEDs are examined. Device A with double EMLs is configured as ITO (120 nm)/TCTA (40 nm)/ TCTA 50 wt $\%$ : BP4mPy 50 wt $\%$ (29 nm)/26DCzPPy $(3 \mathrm{~nm}) / \mathrm{m}^{-}$ MTDATA 10 wt\% : B3PyMPM 90 wt\% (3 nm)/TmPyPB (50 nm)/LiF $(0.8 \mathrm{~nm}) / \mathrm{Al}(150 \mathrm{~nm})$, for which $\mathrm{LiF}$ and aluminum are respectively used as the electron injection layer and reflective cathode. The first EML consisting of TCTA $50 \mathrm{wt} \%$ : BP4mPy $50 \mathrm{wt} \%$ is responsible for the blue emission, while the second EML for the red light was set as $m$-MTDATA $10 \mathrm{wt} \%$ : В3РyMPM $90 \mathrm{wt} \%$. Moreover, the mixing ratio of the aforementioned exciplex-based EMLs were fine-tuned to generate an appropriate white light emission. The device architecture of devices B1 and B2 with triple EMLs consisted of ITO (120 nm)/TCTA (40 nm)/TCTA 50 wt\%: BP4mPy $50 \mathrm{wt} \%$ (29 nm)/m-MTDATA $80 \mathrm{wt} \%$ : TmPyPB $20 \mathrm{wt} \%$ $(x \mathrm{~nm}) / 26 \mathrm{DCzPPy}(3 \mathrm{~nm}) / m$-MTDATA $10 \mathrm{wt} \%$ : В3РyMPM $90 \mathrm{wt} \%$ $(3 \mathrm{~nm}) / \mathrm{TmPyPB}(50 \mathrm{~nm}) / \mathrm{LiF}(0.8 \mathrm{~nm}) / \mathrm{Al}(150 \mathrm{~nm})$. Compared to the architecture of device A, a green EML consisting of $m$-MTDATA $80 \mathrm{wt} \%$ : TmPyPB $20 \mathrm{wt} \%$ was inserted into the device next to the blue EMLs to improve the color rendering capability of the WOLEDs. ${ }^{29}$ The thicknesses of the green EMLs were varied to adjust the emission colors, e.g., $1 \mathrm{~nm}$ for device B1 and $3 \mathrm{~nm}$ for the second set of device B2. The corresponding chemical structures of the materials and the schematic structure of the device architectures are depicted in Fig. 1. 


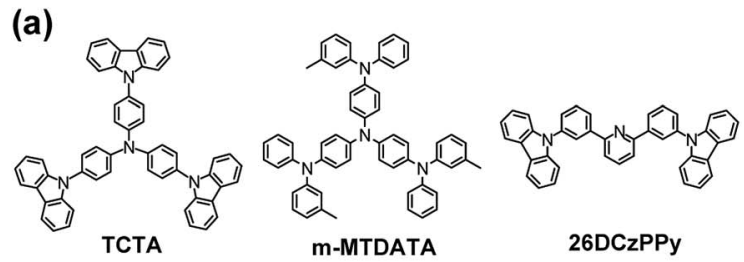

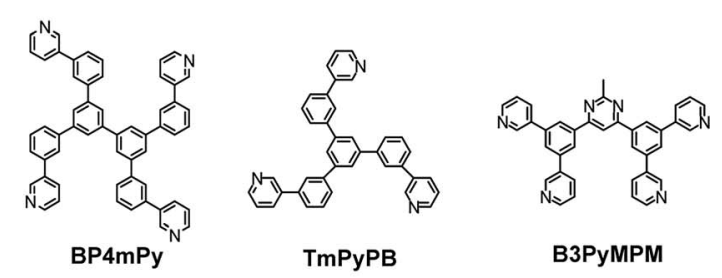

(b)

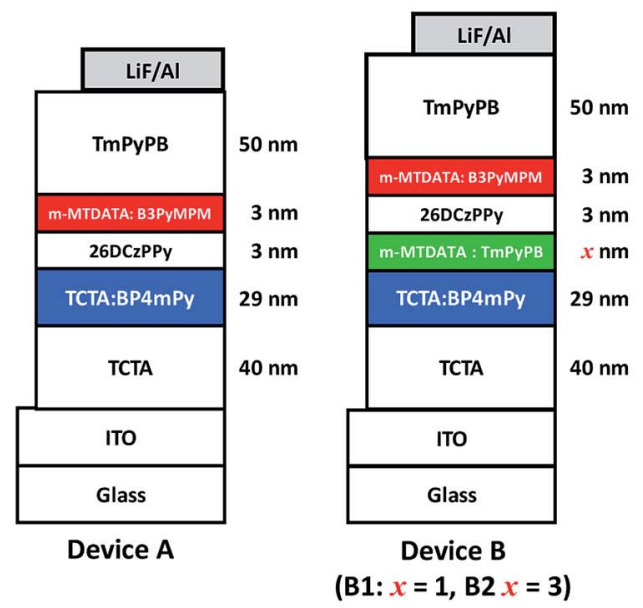

Fig. 1 (a) Chemical structures of the materials; (b) the schematic structure of the device architectures.

Fig. 2 shows the normalized EL spectra and the corresponding CIE coordinates of the tested exciplex-based OLEDs under different biases. From Fig. 2(a), device A exhibited a strong red emission at the lower biases and the blue emission peaks around $440 \mathrm{~nm}$, which increased with the bias. ${ }^{30}$ However, the blue component was still much weaker than the red one even at higher voltages, resulting in device A's emission presenting a pinkish white color ( $c f$. CIE coordinates shown in Fig. 2(d)). On the other hand, the EL spectra of both B1 and B2 showed three peaks with satisfactory emission color. The wavelengths of the deep-blue, sky-blue, and red emission peaks were respectively recorded at $385 \mathrm{~nm}, 462 \mathrm{~nm}$, and $652 \mathrm{~nm}$. Although devices B1 and B2 presented similarly intense red emissions at lower biases, and the emissions ranging from blue to green region gradually increased with voltage, the intensity of the emission ranging blue to green rose significantly at higher voltages, as shown in Fig. 2(b) and (c). Furthermore, the CIE coordinates of both devices B1 and B2 displayed pure white emission at higher biases. The varied emission of device B2 was close to the Planckian locus as the biases increased, indicating the sophisticated design of device B2. In addition, device B2 exhibited high CRI values of about 96 in a wide luminance range from 100 to $1000 \mathrm{~cd} \mathrm{~m}^{-2}$, corresponding to the CCT between 4359 and $7115 \mathrm{~K}$. The numeric data collected from the EL
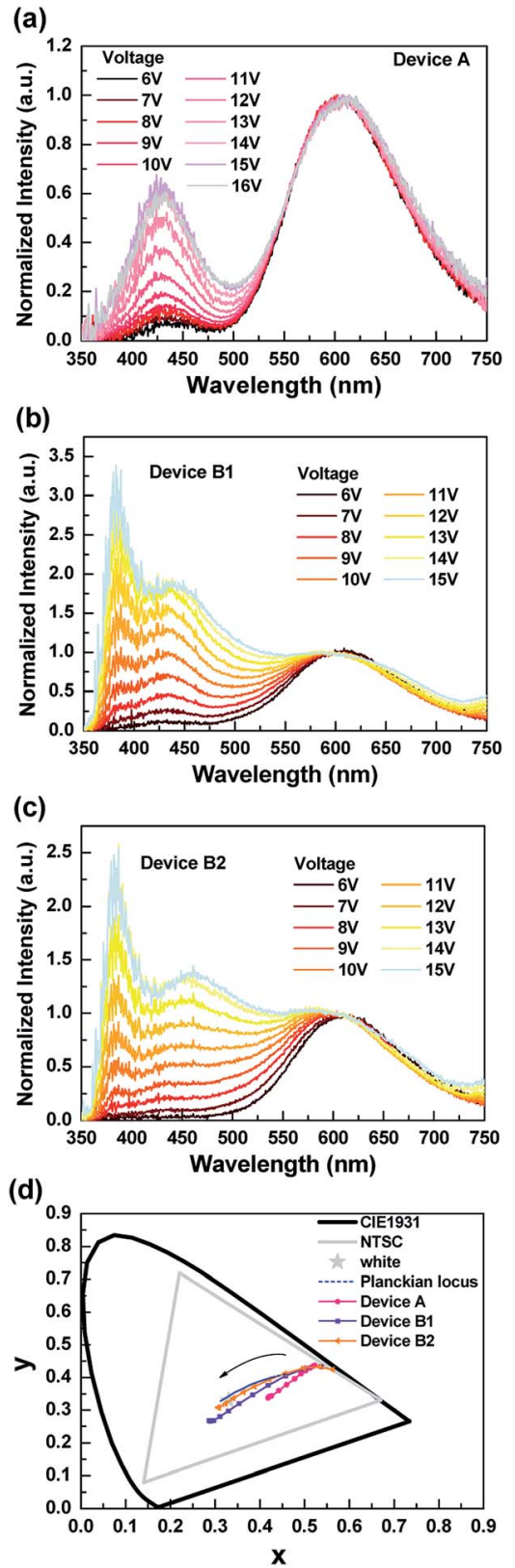

Fig. 2 EL spectra of the tested exciplex-based white OLEDs with different operating voltages. (a) Device A; (b) device B1; (c) device B2; (d) CIE coordinates.

spectra of device B2 are summarized in Table 1. We also included the corresponding data of devices A and B1 in Table S1 in $\mathrm{ESI} \dagger$ for comparison. It's notable that the CRI value of device B2 reached the highest level compared to the previous reports. ${ }^{18}$ These performances demonstrate that this exciplex-based WOLED (i.e. B2) possessed a high-quality white-light illumination. ${ }^{28,31-35}$

Fig. 3 shows the schematic energy level diagram of the tested OLEDs. Given the large barrier at the BЗРуMPM/ 26DCzPPy interface, the hole injection was smoother than the electron injection in device A. As indicated, the red component dominated the EL emission even as the voltage 
Table 1 The numeric data collected from the EL spectra of device B2

\begin{tabular}{lllll}
\hline $\begin{array}{l}\text { Voltage } \\
(\mathrm{V})\end{array}$ & $\begin{array}{l}\text { Luminance } \\
\left(\mathrm{cd} \mathrm{m}^{-2}\right)\end{array}$ & $\begin{array}{l}\text { CIE coordinates } \\
(x, y)\end{array}$ & $\begin{array}{l}\mathrm{CCT} \\
(\mathrm{K})\end{array}$ & CRI \\
\hline 6 & 1.76 & $0.533,0.433$ & 2071 & 70.20 \\
7 & 5.41 & $0.497,0.429$ & 2364 & 75.66 \\
8 & 15.13 & $0.456,0.412$ & 2762 & 81.05 \\
9 & 36.37 & $0.421,0.393$ & 3201 & 85.77 \\
10 & 79.99 & $0.388,0.372$ & 3744 & 89.71 \\
11 & 168.75 & $0.363,0.354$ & 4359 & 92.80 \\
12 & 302.37 & $0.341,0.336$ & 5162 & 95.06 \\
13 & 493.91 & $0.323,0.320$ & 5998 & 96.07 \\
14 & 720.35 & $0.309,0.308$ & 6972 & 96.05 \\
15 & 914.58 & $0.306,0.307$ & 7115 & 95.69 \\
\hline
\end{tabular}
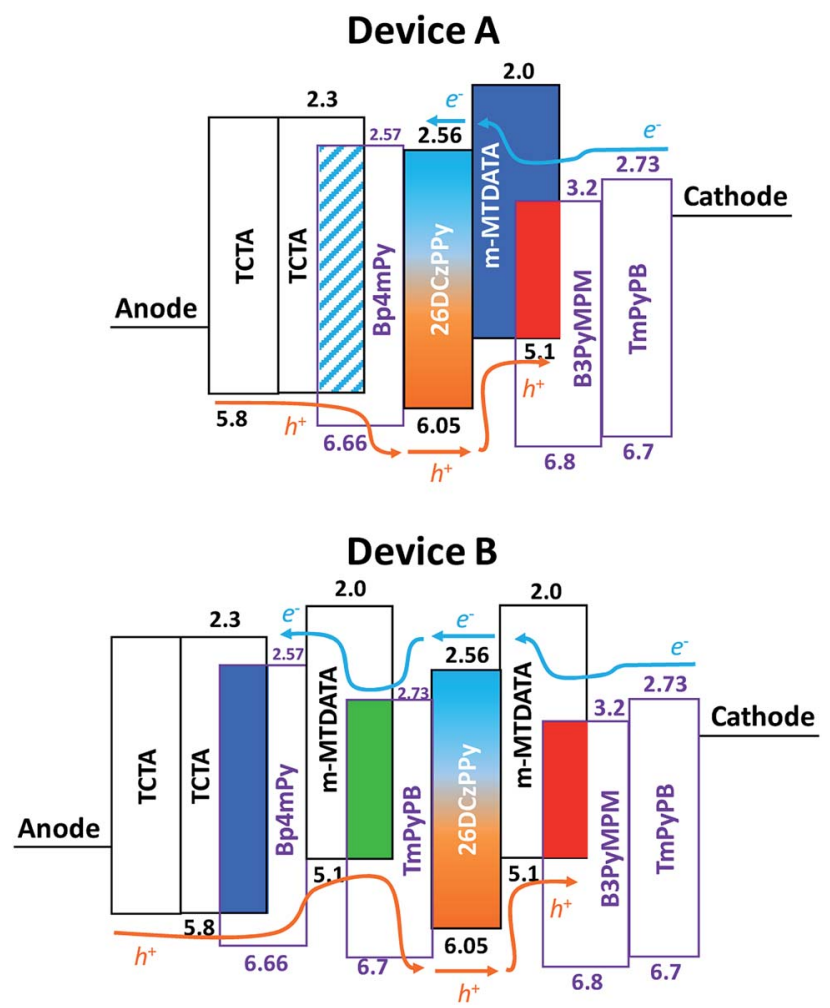

Fig. 3 The schematic energy level diagram of devices A and B.

increased. In addition, abundant hole injection reinforced the carrier recombination occurring in $m$-MTDATA : B3РyMPM layer, increasing the possibility of exciton formation on $\mathrm{m}$ MTDATA molecules. The blue emission peak around $430 \mathrm{~nm}$ should originate from $m$-MTDATA, which could be confirmed by the PL spectrum and will be discussed later (cf. Fig. 5(a)). Consequently, the original setting of blue EML (i.e. TCTA : BP4mPy) in device A was useless. In contrast, for the green EML inserted into devices B1 and B2, the hole would be partially trapped because of the low HOMO level of $\mathrm{m}$ MTDATA, enabling appropriate adjustment of hole injection into the red EML. Thus, the electron could be transported to the green and blue EMLs and then recombine at higher voltages, creating much stronger green and blue emissions.
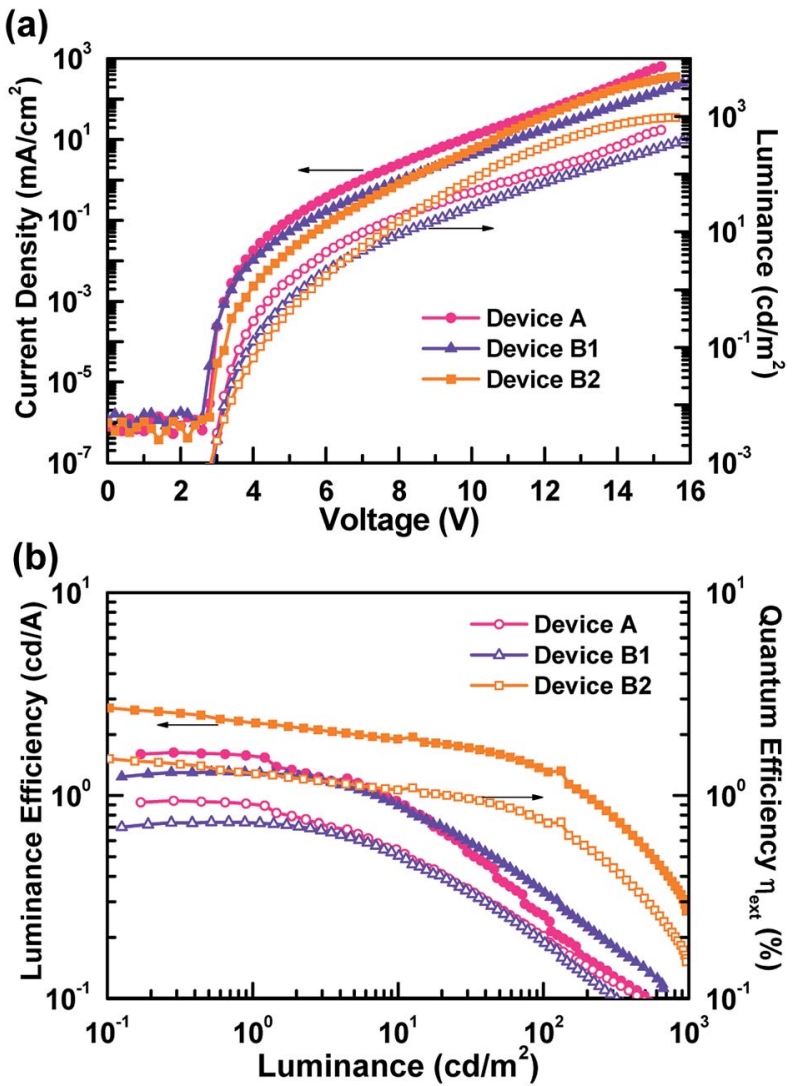

Fig. 4 EL characteristics of the tested exciplex-based white OLEDs: (a) current density-voltage-luminance characteristics, (b) luminance efficiency vs. luminance of devices A, B1, and B2.

Furthermore, by increasing the thickness of the green EML, the emission of device $\mathrm{B} 2$ could be adjusted to present a pure white color. As indicated, the corresponding CIE coordinates of device B2 vary along the Planckian locus as the biases increase.

Fig. 4 and $\mathrm{S} 1 \dagger$ respectively depict the current densityvoltage-luminance $(J-V-L)$ curves and efficiency curves. From Fig. 4(a), as expected, devices B1 and B2 possesses additional green EML, showing lower current densities. ${ }^{36}$ In addition, the current density decreased as the thicknesses of the green EML increased. In contrast, the luminance of device B2 exhibited a higher maximum luminance than the other devices, due to human eye to green..$^{37}$ The efficiency curves shown in Fig. 4(b) and $\mathrm{S} 1 \uparrow$ clearly indicate that a higher carrier balance could be obtained in device B2. The peak external quantum efficiency, luminance efficiency and power efficiency of device B2 were respectively $1.5 \%, 2.7 \mathrm{~cd} \mathrm{~A}^{-1}$, and $2.0 \mathrm{~lm} \mathrm{~W}^{-1}$. This WOLED efficiency was produced using commercially available materials to generate the exciplex. Although the efficiency was much lower than other state-of-art devices, this article focuses on the design of a feasible device architecture for exciplexbased WOLEDs to imitate daylight emissions. Moreover, device efficiency is expected to improve further by replacing the potential HTM/ETM combinations to achieving efficient increased efficiency as well the exciplex emissions. ${ }^{6,8,9,11,12,21}$ 

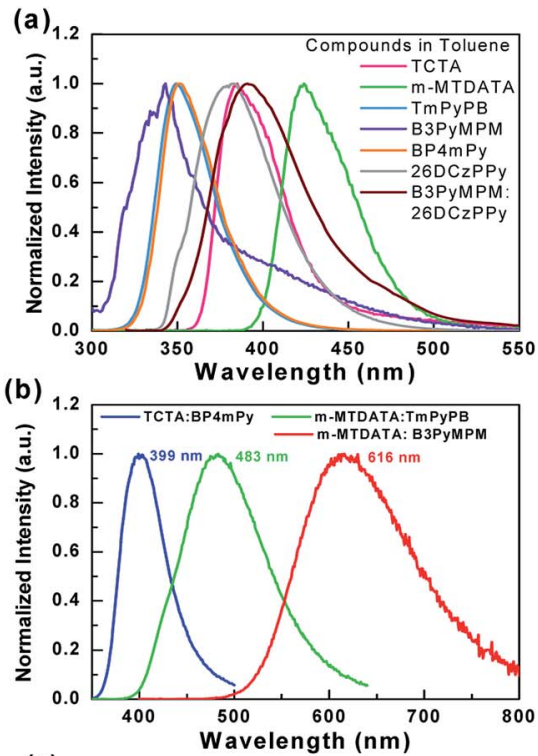

(c)

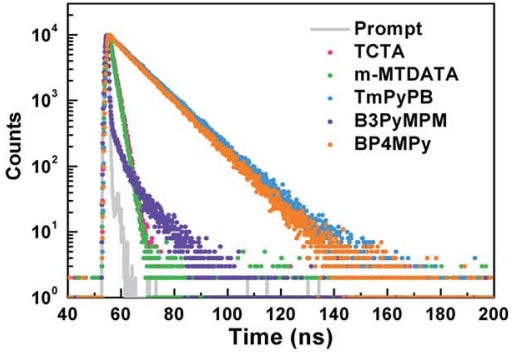

(d)

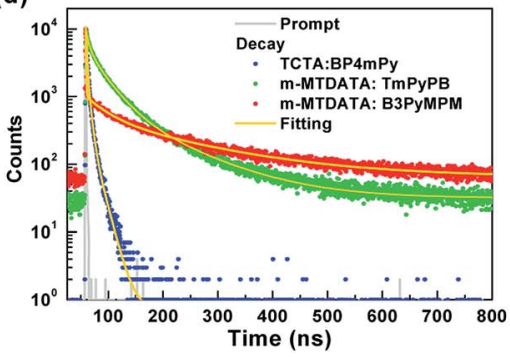

Fig. 5 (a) PL spectra of the materials measured in toluene solution. (b) PL spectra of samples A, B, and C. (c) The decay characteristics of the materials in toluene solution. (d) The decay characteristics of samples A, B, and C.

\section{Photophysical properties of the tested materials and the exciplex films}

Fig. 5 shows the photoluminescence (PL) spectra and the corresponding excited-state lifetime measurements of the used materials. In addition, three thin-film samples composed of the used HTM/ETM mixture were also selected to examine the exciplex formation. The blending ratios of the three samples were the same as those used in the device. Samples B, G, and R respectively represent TCTA $50 \mathrm{wt} \%$ : BP4mPy $50 \mathrm{wt} \%, m^{-}$ MTDATA 80 wt $\%$ : TmPyPB 20 wt $\%, m$-MTDATA 10 wt $\%$ : B3РyMPM $90 \mathrm{wt} \%$. Fig. 5(a) presents the PL spectra of the HTM and ETM measured in toluene at room temperature, while the PL spectra of samples A, B, and C are depicted in Fig. 5(b). Comparing Fig. 2(a) with Fig. 5(a), the emission peak around (a)

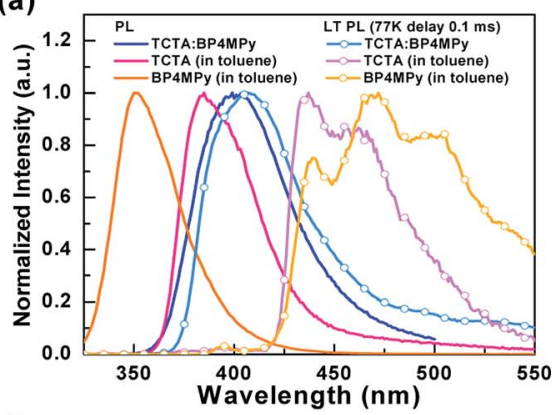

(b)
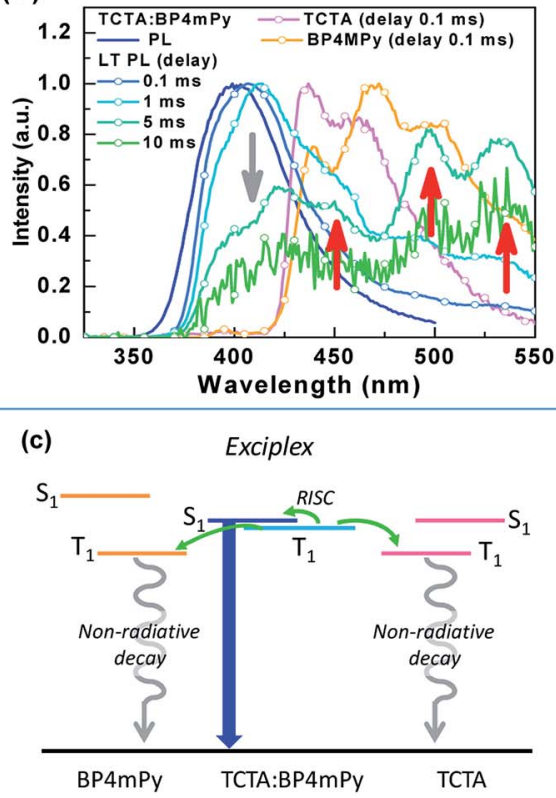

$\mathrm{S}_{1}$

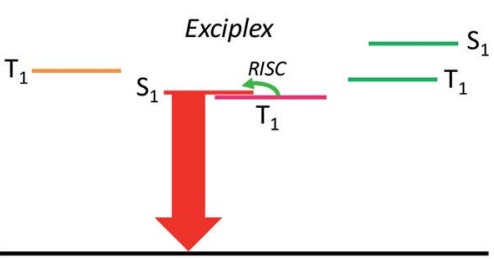

B3PYMPM m-MTDATA:B3PYMPM m-MTDATA

Fig. 6 (a) Fluorescent and phosphorescent spectra of TCTA, BP4mPy, and sample B. (b) Phosphorescent spectra of sample B with different delay times. (c) The schematic diagram of energy transfers in samples $B$ and $\mathrm{R}$.

$430 \mathrm{~nm}$ shown in the EL spectra of device A should originate from $m$-MTDATA. As indicated, the PL spectra of the three samples were akin to the EL spectra of devices B1 and B2, confirming that the EL emission was the origin of the HTM/ ETM mixtures. Furthermore, the emission peaks of all three samples were red-shifted as compared to the corresponding fluorescence of the used materials. ${ }^{38}$ Moreover, the respective full width at half maximum (FWFM) of the three samples were respectively estimated as 56, 106, and $142 \mathrm{~nm}$. Evidently, the FWHM values of samples $G$ and $R$ were much wider than the fluorescence of the common organic emitters, which clearly 
Table 2 The photophysical properties of the used materials

\begin{tabular}{|c|c|c|c|c|c|c|}
\hline \multirow[b]{2}{*}{ Materials } & \multirow[b]{2}{*}{$\lambda_{\text {max. }}(\mathrm{nm})$} & \multirow[b]{2}{*}{ FWHM (nm) } & \multirow[b]{2}{*}{$\mathrm{S}_{1}^{a}(\mathrm{eV})$} & \multirow[b]{2}{*}{$\mathrm{T}_{1}(\mathrm{eV})$} & \multicolumn{2}{|l|}{ Lifetime } \\
\hline & & & & & Prompt (ns) & Decay (ns) \\
\hline $\mathrm{BP} 4 \mathrm{mPy}$ & 351 & 37 & 3.77 & $2.94^{b}$ & 10.6 & n.d. \\
\hline$m$-MTDATA & 424 & 46 & 3.11 & $2.65^{c}$ & 1.8 & n.d. \\
\hline TmPyPB & 349 & 38 & 3.29 & $2.75^{c}$ & 11.3 & n.d. \\
\hline$m$-MTDATA : TmРyPB $(80: 20)$ & 483 & 106 & 3.08 & $3.03^{b}$ & 27.5 & 105.1 \\
\hline$m$-MTDATA : В3РуМРМ $(10: 90)$ & 616 & 142 & 2.37 & $2.36^{b}$ & 28.2 & 175.7 \\
\hline
\end{tabular}

${ }^{a}$ Estimated from the onset of the fluorescent spectrum. ${ }^{b}$ Evaluated from the onset of the phosphorescent spectrum. ${ }^{c}$ Collected from the previous reports (ref. 17, 20 and 21).

demonstrated the exciplex formation..$^{28,39,40}$ However, sample B did not present a typical exciplex emission with a broad spectral profile, but rather exhibited a narrower spectrum than those of samples $\mathrm{G}$ and $\mathrm{R}$.

Fig. 5(c) shows the excited state decay characteristics of the materials in toluene solution. All materials exhibited typical fluorescent decay behavior. On the other hand, the curves exhibited in Fig. 5(d) clearly indicate that samples $\mathrm{G}$ and $\mathrm{R}$ possess two-component decay including a nanosecond-scale prompt and sub-microsecond-scale delayed components, while sample B had only a nanosecond-scale component. The delayed lifetimes for samples $\mathrm{G}$ and $\mathrm{R}$ were respectively estimated at 105.1 and 175.7 ns due to the slower reverse intersystem crossing processes from the $T_{1}$ back to the $S_{1}$ excited state. ${ }^{\mathbf{4 0}}$ Moreover, the respective lifetime values of samples $\mathrm{B}, \mathrm{G}$, and $\mathrm{R}$ were estimated to be $5.3,27.5$, and $28.2 \mathrm{~ns}$ attributed to representative fluorescence decay.

From Fig. 5(b) and (d), sample B seems to exhibit the behavior typical of a fluorescent emitter. However, sample B shows a red-shifted spectrum compared to the fluorescence of TCTA and BP4mPy, suggesting that the emission origin of sample $B$ is different from the $S_{1}$ of TCTA or BP4mPy. Consequently, the phosphorescence of TCTA, BP4mPy, and sample B were measured in $77 \mathrm{~K}$, as shown in Fig. 6(a). Both TCTA and BP4mPy possessed lower triplet energy bandgaps than that of sample B (i.e., TCTA : BP4mpy mixture). The triplet energy bandgaps of TCTA, BP4mPy, and sample B were evaluated based on the onset of the phosphorescent spectra and were respectively estimated to be $2.92,2.94$, and $3.31 \mathrm{eV}$. In addition, the phosphorescence of sample $\mathrm{B}$ was slightly red-shifted compared to the fluorescence. The small energy difference between the single and triplet excited states $\left(\Delta E_{\mathrm{ST}} \sim 0.1 \mathrm{eV}\right)$ confirmed that the emission originated from the exciplex. ${ }^{7,9,39}$ Therefore, the related lower $\mathrm{T}_{1}$ states of TCTA and BP4mPy would offer pathways for exothermic energy transfer, leading to energy loss and thus a shorter lifetime for sample B. The photophysical properties of the used materials and the thin-film samples were summarized in Table 2. Furthermore, we measured the phosphorescence of sample B with different delay times, as shown in Fig. 6(b). The phosphorescence of exciplex gradually decayed with the extending delay time, while additional emissions appeared consisting of peaks similar to the BP4mPy's phosphorescence. These additional emissions could be seen as direct evidence of exothermic energy transfer. This was the first demonstration of the assumption indicating that the selected HTM and ETM should possess higher triplet energy bandgaps than those of their corresponding exciplex to avoid energy loss to the composed single molecules. ${ }^{41}$ Fig. 6(c) shows a schematic diagram of the energy transfers in samples $B$ and $\mathrm{R}$.

\section{Conclusions}

In conclusion, we propose a specific device architecture for WOLEDs without any molecular emitter, realizing a pure exciplex emission generated by the mixed HTMs/ETMs layers. Each red, green, and blue emission could be obtained by combining the appropriate HTM and ETM. Furthermore, a bipolar buffer layer was inserted between the green and red EMLs to adjust the carrier distribution and thus alter the emission. As a result, the corresponding CIE coordinates varies along with the Planckian locus as bias increases, realizing that WOLEDs imitate daylight. Furthermore, photophysical results indicate that the HTM/ETM used to produce exciplex should possess a higher triplet energy bandgap than that of the exciplex, thus avoiding energy loss to the composed single molecules.

\section{Conflicts of interest}

The authors declare no conflict of interest.

\section{Acknowledgements}

The authors gratefully acknowledge financial support from the Ministry of Science and Technology of Taiwan. (MOST 106-2221E-155-035).

\section{References}

1 A. Endo, M. Ogasawara, A. Takahashi, D. Yokoyama, Y. Kato and C. Adachi, Adv. Mater., 2009, 21, 4802. 
2 A. Endo, K. Sato, K. Yoshimura, T. Kai, A. Kawada, H. Miyazaki and C. Adachi, Appl. Phys. Lett., 2011, 98, 083302.

3 H. Uoyama, K. Goushi, K. Shizu, H. Nomura and C. Adachi, Nature, 2012, 492, 234.

4 K. Goushi, K. Yoshida, K. Sato and C. Adachi, Nat. Photonics, 2012, 6, 253.

5 W.-Y. Hung, G.-C. Fang, Y.-C. Chang, T.-Y. Kuo, P.-T. Chou, S.-W. Lin and K.-T. Wong, ACS Appl. Mater. Interfaces, 2013, 5, 6826.

6 W.-Y. Hung, P.-Y. Chiang, S.-W. Lin, W.-C. Tang, Y.-T. Chen, S.-H. Liu, P.-T. Chou, Y.-T. Hung and K.-T. Wong, ACS Appl. Mater. Interfaces, 2016, 8, 4811.

$7 \mathrm{~J} . \mathrm{Li}, \mathrm{H}$. Nomura, H. Miyazaki and C. Adachi, Chem. Commun., 2014, 50, 6174.

8 X.-K. Liu, Z. Chen, C.-J. Zhang, C.-L. Liu, C.-S. Lee, F. Li, X.-M. Ou and X.-H. Zhang, Adv. Mater., 2015, 27, 2378.

9 W. Liu, J.-X. Chen, C.-J. Zheng, K. Wang, D.-Y. Chen, F. Li, Y.-P. Dong, C.-S. Lee, X.-M. Ou and X.-H. Zhang, Adv. Funct. Mater., 2016, 26, 2002.

10 S.-J. He, D.-K. Wang, N. Jiang, J. S. Tse and Z.-H. Lu, Adv. Mater., 2016, 28, 649.

11 K.-H. Kim, S.-J. Yoo and J.-J. Kim, Chem. Mater., 2016, 28, 1936.

12 W.-Y. Hung, T.-C. Wang, P.-Y. Chiang, B.-J. Peng and K.-T. Wong, ACS Appl. Mater. Interfaces, 2017, 9, 7355.

13 D. Luo, Y. Xiao, M. Hao, Y. Yang, Y. Gao and B. Liu, Appl. Phys. Lett., 2017, 110, 061105.

14 B. Liu, H. Nie, X. Zhou, S. Hu, D. Luo, D. Gao, J. Zou, M. Xu, L. Wang, Z. Zhao, A. Qin, J. Peng, H. Ning, Y. Cao and B.-Z. Tang, Adv. Funct. Mater., 2016, 26, 776.

15 B. Liu, H. Tao, L. Wang, D. Gao, W. Liu, J. Zou, M. Xu, H. Ning, J. Peng and Y. Cao, Nano Energy, 2016, 26, 26.

16 B. Liu, H. Nie, G. Lin, S. Hu, D. Gao, J. Zou, M. Xu, L. Wang, Z. Zhao, H. Ning, J. Peng, Y. Cao and B.-Z. Tang, ACS Appl. Mater. Interfaces, 2017, 9, 34162.

17 J.-H. Jou, M.-H. Wu, S.-M. Shen, H.-C. Wang, S.-Z. Chen, S.-H. Chen, C.-R. Lin and Y.-L. Hsieh, Appl. Phys. Lett., 2009, 95, 013307.

18 J.-H. Jou, C.-Y. Hsieh, J.-R. Tseng, S.-H. Peng, Y.-C. Jou, J. H. Hong, S.-M. Shen, M.-C. Tang, P.-C. Chen and C.-H. Lin, Adv. Funct. Mater., 2013, 23, 2750.

19 J.-H. Jou, R.-Z. Wu, H.-H. Yu, C.-J. Li, Y.-C. Jou, S.-H. Peng, Y.-L. Chen, C.-T. Chen, S.-M. Shen, P. Joers and C.-Y. Hsieh, ACS Photonics, 2014, 1, 27.

20 D. Luo, X.-L. Li, Y. Zhao, Y. Gao and B. Liu, ACS Photonics, 2017, 4, 1566.

21 D. Luo, Q. Chen, Y. Gao, M. Zhang and B. Liu, ACS Energy Lett., 2018, 3, 1531.
22 M. Huang, B. Jiang, G. Xie and C. Yang, J. Phys. Chem. Lett., 2017, 8, 4967.

23 W. Song, H.-L. Lee and J.-Y. Lee, J. Mater. Chem. C, 2017, 5, 5923.

24 S.-J. Su, D. Tanaka, Y.-j. Li, H. Sasabe, T. Takeda and J. Kido, Org. Lett., 2008, 10, 941.

25 S.-J. Su, Y. Takahashi, T. Chiba, T. Takeda and J. Kido, Adv. Funct. Mater., 2009, 19, 1260.

26 J.-H. Lee, S.-H. Cheng, S.-J. Yoo, H. Shin, J.-H. Chang, C.-I. Wu, K.-T. Wong and J.-J. Kim, Adv. Funct. Mater., 2015, 25, 361.

27 S.-J. Su, H. Sasabe, T. Takeda and J. kido, Chem. Mater., 2008, 20, 1691.

28 B. Zhao, H. Zhang, Y. Miao, Z. Wang, L. Gao, H. Wang, Y. Hao and W. Li, J. Mater. Chem. C, 2018, 6, 304.

29 S. Wang, X. Wang, B. Yao, B. Zhang, J. Ding, Z. Xie and L. Wang, Sci. Rep., 2015, 5, 12487.

30 E. Angioni, M. Chapran, K. Ivaniuk, N. Kostiv, V. Cherpak, P. Stakhira, A. Lazauskas, S. Tamulevicius, D. Volyniuk, N. J. Findlay, T. Tuttle, J. V. Grazulevicius and P. J. Skabara, J. Mater. Chem. C, 2016, 4, 3851.

31 C.-H. Chang, C.-C. Chen, C.-C. Wu, S.-Y. Chang, J.-Y. Hung and Y. Chi, Org. Electron., 2010, 11, 266.

32 C.-H. Chang, K.-C. Tien, C.-C. Chen, M.-S. Lin, H.-C. Cheng, S.-H. Liu, C.-C. Wu, J.-Y. Hung, Y.-C. Chiu and Y. Chi, Org. Electron., 2010, 11, 412.

33 B. Liu, M. Xu, L. Wang, H. Tao, Y. Su, D. Gao, L. Lan, J. Zou and J. Peng, Nano-Micro Lett., 2014, 6, 335.

34 B. Liu, D. Luo, J. Zou, D. Gao, H. Ning, L. Wang, J. Peng and Y. Cao, J. Mater. Chem. C, 2015, 3, 6359.

35 B. Liu, X.-L. Li, H. Tao, J. Zou, M. Xu, L. Wang, J. Peng and Y. Cao, J. Mater. Chem. C, 2017, 5, 7668.

36 S. Hofmann, M. Furno, B. Lussem, K. Leo and M. C. Gather, Phys. Status Solidi A, 2013, 8, 1467.

37 D.-G. Chen, T.-C. Lin, C.-L. Chen, Y.-T. Chen, Y.-A. Chen, G.-H. Lee, P.-T. Chou, C.-W. Liao, P.-C. Chiu, C.-H. Chang, Y.-J. Lien and Y. Chi, ACS Appl. Mater. Interfaces, 2018, 10, 12886.

38 C.-H. Chang, S.-W. Wu, C.-W. Huang, C.-T. Hsieh, S.-E. Lin, N.-P. Chen and H.-H. Chang, Jpn. J. Appl. Phys., 2017, 56, 129209.

39 T. Lin, T. Zhang, Q. Song, F. Jin, Z. Liu, Z. Su, Y. Luo, B. Chu, C. S. Lee and W. Li, Org. Electron., 2016, 38, 69.

40 S. Yuan, X. Du, J. Zhao, W. Liu, H. Lin, C. Zheng, S. Tao and X. Zhang, Org. Electron., 2016, 39, 10.

41 H. Lim, H. Shin, K.-H. Kim, S.-J. Yoo, J.-S. Huh and J.-J. Kim, ACS Appl. Mater. Interfaces, 2017, 9, 37883. 